\title{
Interview - Sicherer für den Patienten, praktikabler im klinischen Alltag
}

Kardiologen und Radiologen veröffentlichen das weltweit erste Konsensuspapier zur Durchführung von MRT-Untersuchungen bei Patienten mit aktiven kardialen Implantaten.

Berlin, 9.2.2017. Die Deutsche Gesellschaft für Kardiologie (DGK) und die Deutsche Röntgengesellschaft (DRG) haben gemeinsam das Konsensuspapier „MRT-Untersuchungen bei Patienten mit Herzschrittmachern und implantierbaren Kardioverter-Defibrillatoren“ veröffentlicht. PD Dr. Torsten Sommer, Chefarzt der Abteilung für Diagnostische und Interventionelle Radiologie, Deutsches Rotes Kreuz - Krankenhaus Neuwied und Erstautor, erläutert im Interview die zentralen Inhalte des Papiers und erklärt, weshalb es den Zugang dieser Patientengruppe zu notwendigen MR-Untersuchungen verbessert.

DRG: Wo liegen die klinische Relevanz und die Bedeutung der Thematik des Konsensuspapiers?

Sommer Allein in Deutschland leben aktuell etwa eine Million Patienten mit aktiven kardialen Implantaten, das heißt mit einem Herzschrittmacher oder einem implantierbaren Kardioverter-Defibrillator (ICD). Und die Tendenz steigt, vor allem im ICDBereich, insbesondere durch die demografische Entwicklung. Wenn jedoch eine so große Patientengruppe keinen Zugang zur
Diagnostik per Magnetresonanztomografie (MR) hat, einem Untersuchungsverfahren, das in vielen Fällen die bestmögliche Bildgebung für Diagnostik und Therapieplanung darstellt, führt das zu großen Problemen in der Patientenversorgung. Die gute Nachricht ist: Die Situation hat sich für Schrittmacher- und ICD-Patienten in den letzten Jahren durch intensive internationale Forschungsarbeiten, an denen auch deutsche radiologische Institutionen maßgeblich beteiligt waren, erheblich gebessert: Patienten mit konventionellen Schrittmachern oder ICD können unter dezidierten Sicherheitsvorkehrungen als „off label use" untersucht werden, und vor allem sind jetzt sogenannte bedingt MR-sichere Schrittmacher und ICDs verfügbar, die unter bestimmten definierten Rahmenbedingungen zertifiziert und zugelassen sind.

\section{Das klingt positiv. Aber wo ist der $\mathrm{Ha}$ -} ken?

Das Problem liegt darin, dass die meisten Schrittmacher- und ICD-Patienten - selbst, wenn sie mit der neuen, bedingt MR-sicheren Technologie versorgt wurden - leider immer noch große Schwierigkeiten haben, eine MR-Untersuchung zu erhalten. Die Hauptgründe hierfür sind der Informationsmangel bezüglich dieser neuen und komplexen Thematik bei Zuweisern, Kardiologen und Radiologen, aber auch die Unklarheiten über konkrete Handlungsabläufe und Verantwortlichkeiten in der

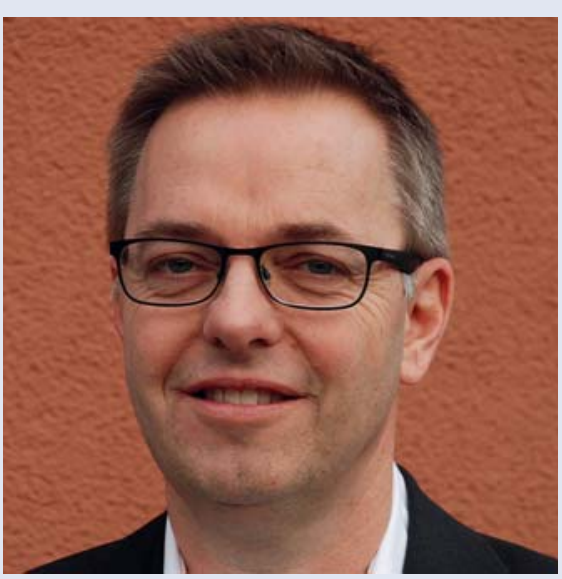

PD Dr. Torsten Sommer

Zusammenarbeit zwischen Radiologen und Kardiologen.

Nun gibt es ja zu diesem Thema schon Veröffentlichungen der einzelnen Fachgesellschaften: die Leitlinien der Europäischen Gesellschaft für Kardiologie (ESC) aus dem Jahr 2013 und das Positionspapier der DRG von 2015. Wie ist vor diesem Hintergrund das Konsensuspapier von DGK und DRG einzuordnen?

Ich habe grundsätzlich großen Respekt vor kardiologischen Leitlinien. In dem entsprechenden Kapitel der ESC-Publikation allerdings wird die ganze Thematik mit stark vereinfachenden Handlungsalgorithmen verkürzt auf knapp zwei Seiten dargestellt, die der Komplexität des Themas nicht 
gerecht werden. Es fehlen beispielsweise wichtige sicherheitsrelevante Aspekte wie die pulsoxymetrische Patientenüberwachung und praktisch alle MR-technisch relevanten und beinflussbaren Sicherheitsaspekte zur Risikominimierung. Dazu gehört unter anderem die Möglichkeit der Reduktion der eingestrahlten Hochfrequenzenergie durch entsprechende Modifikationen der MR-Sequenzparameter.

\section{Und welches Ziel hatte das DRG-Papier von 2015?}

Das DRG-Positionspapier von 2015 war eine Reaktion auf die ESC-Publikation. Es hat die Thematik von MRT-Untersuchungen bei Schrittmacherpatienten aus radiologischer und MR-physikalischer Perspektive ausführlich dargestellt und die Inhalte des ESCPapiers konkretisiert, ergänzt und in einigen Punkten auch korrigiert. Die logische Weiterentwicklung dieser beiden Publikationen, die von beiden deutschen Fachgesellschaften gleichermaßen angestrebt wurde, ist das jetzt publizierte gemeinsame Konsensuspapier der Deutschen Gesellschaft für Kardiologie und der Deutschen Röntgengesellschaft. Hier wird ein abgestimmtes und von beiden Fachgesellschaften ratifiziertes Vorgehen vorgestellt, das insbesondere an den Schnittstellen der Zusammenarbeit zwischen Radiologie und Kardiologie in den Bereichen Indikationsstellung, Risikoabschätzung und Patientenüberwachung klare Inhalte und Verant- wortlichkeiten definiert. Das Papier stellt außerdem dezidierte Umprogrammierungsoptionen der Schrittmacher- und ICD-Systeme vor, die bezüglich Patientensicherheit und Praktikabilität im klinischen Alltag optimiert sind.

\section{Wie gestaltete sich die Zusammenarbeit} mit den Kollegen aus der Kardiologie?

Der Anfang der Zusammenarbeit war nicht gänzlich spannungsfrei und von kleineren berufspolitischen Revierscharmützeln und einigen robusten E-Mails durchzogen. Mit der Zeit - und über die Diskussion von Sachfragen und dem Zusammenführen der verschiedenen Fachexpertisen aus Kardiologie, Elektrophysiologie, MR-Physik und Radiologie - entwickelte sich dann eine sehr gute, kollegiale und fruchtbare Zusammenarbeit. Das Autorenteam freut sich jedenfalls, dass es dieses Konsensuspapier nach zwei Jahren Arbeit und der Passage von insgesamt fünf zustimmungspflichtigen Gremien letztlich ohne relevante Friktionen oder inhaltliche Abstriche zu einem Abschluss führen konnte!

Das Konsensuspapier ist ein wichtiger Schritt für beide Fachdisziplinen. Welche Bedeutung hat das Papier, und welche Ziele verfolgt es?

Es ist der weltweit erste publizierte Konsensus zwischen einer radiologischen und einer kardiologischen nationalen Fachgesellschaft mit abgestimmten konkreten Hand- lungsempfehlungen zum prozeduralen Management von MRT-Untersuchungen bei Schrittmacher- und ICD-Patienten. Ziel dieses Konsensuspapiers ist es, einerseits natürlich ein Maximum an Patientensicherheit zu gewährleisten, und anderseits ein rechtssicheres, praktikables und im klinischen Alltag realisierbares Vorgehen zu etablieren, um eine breitere und bessere MR-Versorgung der Schrittmacher- und ICD-Patienten, auch außerhalb spezialisierter Institutionen, zu erreichen.

Sind aktuell neue technische Entwicklungen auf diesem Gebiet zu erwarten?

Definitiv. Auf der einen Seite arbeiten die Schrittmacherfirmen konkret an Schrittmacher- und ICD-Systemen mit automatischer Magnetfelddetektion, was die Praktikabilität wegen der Möglichkeit der primär ortsund zeitversetzten Umprogrammierung deutlich erhöhen würde. Auf der anderen Seite leisten jetzt auch die Hersteller von MR-Systemen, die sich bezüglich der Thematik während der letzten zehn Jahre äußerst bedeckt gehalten haben, einen wichtigen Beitrag durch neue SoftwareEntwicklungen und Benutzeroberflächen, die eine bessere Kontrolle und Limitation sicherheitsrelevanter MR-Parameter bei der Untersuchung von Patienten mit aktiven und passiven Implantaten ermöglichen.

Vielen Dank für das Gespräch!

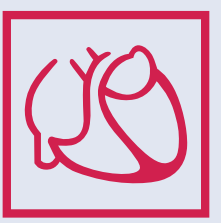

